\title{
OPEN Testosterone therapy masculinizes speech and gender presentation in transgender men
}

\author{
Carolyn R. Hodges-Simeon ${ }^{1,7 凶}$, Graham P. O. Grail ${ }^{1,2,7}$, Graham Albert ${ }^{1}$, Matti D. Groll ${ }^{3,5}$, \\ Cara E. Stepp ${ }^{3,4,5}$, Justin M. Carré6 ${ }^{6}$ Steven A. Arnocky ${ }^{6}$
}

Voice is one of the most noticeably dimorphic traits in humans and plays a central role in gender presentation. Transgender males seeking to align internal identity and external gender expression frequently undergo testosterone $(T)$ therapy to masculinize their voices and other traits. We aimed to determine the importance of changes in vocal masculinity for transgender men and to determine the effectiveness of $T$ therapy at masculinizing three speech parameters: fundamental frequency (i.e., pitch) mean and variation $\left(f_{\mathrm{o}}\right.$ and $f_{\mathrm{o}}-\mathrm{SD}$ ) and estimated vocal tract length (VTL) derived from formant frequencies. Thirty transgender men aged 20 to 40 rated their satisfaction with traits prior to and after $T$ therapy and contributed speech samples and salivary $T$. Similar-aged cisgender men and women contributed speech samples for comparison. We show that transmen viewed voice change as critical to transition success compared to other masculine traits. However, $T$ therapy may not be sufficient to fully masculinize speech: while $f_{\mathrm{o}}$ and $f_{\mathrm{o}}$-SD were largely indistinguishable from cismen, VTL was intermediate between cismen and ciswomen. $f_{0}$ was correlated with salivary $\mathrm{T}$, and VTL associated with $\mathrm{T}$ therapy duration. This argues for additional approaches, such as behavior therapy and/or longer duration of hormone therapy, to improve speech transition.

Transgender individuals describe the incongruence between their assigned sex at birth and their own gender identity to be a significant source of distress ${ }^{1-3}$. Compared to cisgender individuals, trans individuals have higher rates of suicide and suicide attempts ${ }^{4}$, distress ${ }^{5,6}$, depression, and anxiety ${ }^{7}$ and are more likely to be the victims of harassment and violence ${ }^{8}$. As a result, many seek gender confirmation surgeries or testosterone (T) therapy to bring their physical appearance and/or speech into alignment with their experienced gender. These interventions are generally effective: recipients report greater external validation of their gender from social engagements following treatment ${ }^{9}$ as well as overall improvements in quality of life ${ }^{10-12}$. For transgender men (referred to as transmen throughout) undergoing $\mathrm{T}$ therapy, more masculine speech is correlated with greater reported well-being ${ }^{2}$.

Two key acoustic characteristics of speech independently contribute to a masculine-sounding voice ${ }^{13-16}: 1$ ) fundamental frequency $\left(f_{\mathrm{o}}\right)$ of vocal fold vibration, relating to voice pitch, and 2$)$ the spectral structure of speech formants, which give identity to vowels and thus speech content, but also reflect vocal tract length (VTL) ${ }^{17}$. During puberty in cisgender males (whose natal sex and identity are both male; called "cismen" throughout), both the length and thickness of the vocal folds increase alongside T levels, thereby lowering $f_{\mathrm{o}}{ }^{18-24}$. The relationship between $\mathrm{T}$ and $f_{\mathrm{o}}$ remains consistent following puberty such that adult cismen with lower $f_{\mathrm{o}}$ also have higher salivary $\mathrm{T}^{25-28}$ (but not always ${ }^{29}$ ). Compared to ciswomen, cismen's vocal folds are approximately $60 \%$ longer ${ }^{30}$ and speaking $f_{\mathrm{o}}$ is, on average, $80 \mathrm{~Hz}$ lower ${ }^{31}$. In addition to vocal fold changes, the larynx also descends in cismen during puberty, resulting in a larger and longer vocal tract. This $10-20 \%$ difference in VTL between cismen and ciswomen accounts for overall lower formant frequencies in cismen that occupy a smaller total frequency range $\mathrm{e}^{17,24,28,31}$.

In previous studies of $\mathrm{T}$ therapy-related changes in the voices of transmen, $f_{\mathrm{o}}$ is reliably reduced in most participants after sufficient time on T therapy. Longitudinal studies demonstrate significant (e.g., $\sim 60-70 \mathrm{~Hz}$ )

\footnotetext{
${ }^{1}$ Department of Anthropology, Boston University, 232 Bay Stated Rd., Room 102-B, Boston, MA 02215, USA. ${ }^{2}$ Department of Forensic Sciences, George Washington University, Washington, D.C., USA. ${ }^{3}$ Department of Speech, Language, and Hearing Sciences, Boston University, Boston, MA, USA. ${ }^{4}$ Department of Otolaryngology - Head and Neck Surgery, Boston University School of Medicine, Boston, MA, USA. ${ }^{5}$ Department of Biomedical Engineering, Boston University, Boston, MA, USA. ${ }^{6}$ Department of Psychology, Nipissing University, North Bay, ON, Canada. ${ }^{7}$ These authors contributed equally: Carolyn R. Hodges-Simeon and Graham P. O. Grail. ${ }^{\boxplus}$ email: crhodges@bu.edu
} 
and stable within-participant reductions in $f_{\mathrm{o}}$ after a minimum of $\sim 3-4$ months on T therapy ${ }^{32-35}$. Accordingly, participants typically perceived their own voices to be lower in pitch ${ }^{32}$ and participants with lower $f_{\mathrm{o}}$ reported a higher likelihood of being perceived as male over the phone ${ }^{35}$. Although T therapy often plays a significant role in female-to-male transition-noted by $f_{\mathrm{o}}$ values that are largely indistinguishable from those of cismen for many speakers ${ }^{36}$ - not all individuals experience enough $f_{\mathrm{o}}$ lowering to reach the typical range of cismen. A meta-analysis found that $21 \%$ of patients fail to reach the $f_{\mathrm{o}}$ range of cismen after one year on T therapy ${ }^{37}$, by which time voice $f_{\mathrm{o}}$ lowering has typically reached an asymptote regardless of dose regimen ${ }^{38}$. Not surprisingly, an estimated $12-16 \%$ of patients are not fully satisfied with their vocal transition ${ }^{32,37}$.

Fundamental frequency $\left(f_{o}\right)$ is often reported to be the most heavily weighted cue for listeners in determining speaker gender identity ${ }^{39,40}$. However, studies of experimentally manipulated speech, in which $f_{\mathrm{o}}$ and formant frequencies are varied independently, reveal that listeners rely on more than just $f_{\mathrm{o}}$ when making judgments of speaker gender. For example, listeners can correctly identify ciswomen speakers as women even when their $f_{\mathrm{o}}$ values are artificially lowered to the range of cismen ${ }^{41}$. When $f_{\mathrm{o}}$ and formant frequencies are altered to the same perceptual degree (based on empirically derived just-noticeable-differences), listeners appear to rely more on formant information when making judgments about speaker body size, masculinity, and physical dominance ${ }^{28,42}$ ( $\mathrm{cf}$. Hillenbrand \& Clark $^{15}$ ). Thus, speech features other than $f_{\mathrm{o}}$ may impact the externally perceived gender identity of individuals undergoing the female-to-male transition, and these additional features, such as formant frequencies or the related measure of VTL, can potentially be used as outcome measures to assess efficacy of transition strategies and increase patient satisfaction. Yet, aside from one case study ${ }^{43}$ and an unpublished dissertation ${ }^{44}$, no studies on transgender men have investigated changes in formant measures with $\mathrm{T}$ therapy.

The present research has four central research questions. First, how important is masculinization of speech parameters relative to other traits for those undergoing a female-to-male transition? Second, is T therapy effective at masculinizing acoustic properties of speech that drive gender perception: $f_{\mathrm{o}}$ (mean and variation, $f_{\mathrm{o}}-\mathrm{SD}$ ) and formant-based estimates of VTL? Research suggests that both are critical to perception of gender; however, little research exists on formant changes in transmen. Third, do higher salivary $\mathrm{T}$ levels and a longer duration of T therapy contribute to more masculine speech parameters? Finally, how do transmen rate their satisfaction with speech changes compared to other changes that occurred during T therapy?

\section{Results}

How important is masculinization of speech parameters for transmen undergoing $\mathrm{T}$ therapy? Voice masculinity was rated by participants as one of the traits they were least satisfied with prior to transition compared with all other traits (See Fig. 1A); 77\% of participants rated voice masculinity as a "1" or a " 2 " on the 1-to-7 scale ( 1 indicated that they were extremely unhappy with the trait). Furthermore, when asked to rank the importance of observing change relative to other traits, change in voice masculinity was ranked as most important (see Fig. 1B); 83\% of participants ranked it as a "6" or "7" (7 indicated that it was very important to see changes).

Is T therapy effective at masculinizing acoustic properties of speech that drive gender perception? Results suggest that T therapy is effective at masculinizing transmen's $f_{\mathrm{o}}$ and $f_{\mathrm{o}} \mathrm{-SD}$. A univariate ANOVA showed a significant effect of group on $f_{\mathrm{o}}\left[F(2,93)=164.8, p<0.001, \eta^{2}=0.78\right], f_{\mathrm{o}}-\mathrm{SD}[F(2,93)=45.4$, $\left.p<0.001, \eta^{2}=0.49\right]$, and VTL $\left[F(2,92)=47.5, p<0.001, \eta^{2}=0.51\right]$. Post-hoc comparisons using Tukey's HSD showed that the $f_{\mathrm{o}}$ and $f_{\mathrm{o}}-\mathrm{SD}$ of transmen were significantly lower than the $f_{\mathrm{o}}$ and $f_{\mathrm{o}}-\mathrm{SD}$ of ciswomen (Cohen's $d=3.5$ and 1.5 , respectively), while indistinguishable from those of cismen. None of the transmen fell outside the cismen range for $f_{\mathrm{o}}$ (see Table 1 ); however, seven transmen $(23 \%)$ had greater $f_{\mathrm{o}}-\mathrm{SD}$ than the highest value in the cismen group. Transmen's estimated VTL was significantly longer than ciswomen (Cohen's $d=1.0$ ), but shorter than cismen (Cohen's $d=1.4$ ). Further, $23 \%$ of the VTL estimates were smaller in transmen than the lowest value in our cismen sample. See Table 1 for the mean, standard deviation, and range of speech parameters for transmen, cismen, and ciswomen groups. See Fig. 2 for visual comparisons of the three groups.

Do higher salivary $\mathrm{T}$ levels and a longer duration of $\mathrm{T}$ therapy contribute to more masculine speech parameters? Two individuals with very high T levels $(2,889$ and $794 \mathrm{pg} / \mathrm{mL})$ were identified using Grubbs outlier test ${ }^{45}$ and excluded from the following analyses. Salivary T was significantly correlated with lower mean $f_{\mathrm{o}}(r=-0.37, p=0.05)$, but not $f_{\mathrm{o}}-\mathrm{SD}(r=-0.14, n s)$ or VTL $(r=0.03, n s)$. See Fig. 3 . Testosterone levels were significantly correlated with time on $\mathrm{T}$ therapy; individuals who have been on therapy for a longer duration had higher T levels $(r=0.39, p<0.05)$. See Fig. 4 . Therefore, multiple regression models were then used to examine the independent contributions of circulating $\mathrm{T}$ and time on $\mathrm{T}$ therapy to each masculine speech parameter. Salivary T significantly predicted lower $f_{\mathrm{o}}(\beta=-0.46, \mathrm{SE}=14.39, t=-2.23, p=0.04)$, but $\mathrm{T}$ therapy duration did not $(\beta=0.19, \mathrm{SE}=5.48, t=0.90, p=0.35)$. As a second step, we added the age at beginning of $\mathrm{T}$ therapy to the model. Only salivary $\mathrm{T}$ was associated with lower $f_{\mathrm{o}}$; however, it did not reach conventional significance levels $(\beta=-0.38, \mathrm{SE}=14.53, t=-1.83, p=0.08)$. In a separate model predicting VTL, T therapy duration was a significant predictor $(\beta=0.46, \mathrm{SE}=0.25, t=2.30, p=0.03)$, while salivary $\mathrm{T}(\beta=-0.09, \mathrm{SE}=0.67, t=-0.41, p=0.69)$ and age at beginning $\mathrm{T}$ therapy were not $(\beta=0.28, \mathrm{SE}=0.03, t=1.49, p=0.15)$. The model predicting $f_{\mathrm{o}}-\mathrm{SD}$ was not significant.

How do transmen rate their satisfaction with self-perceived speech changes compared to other changes that occurred during T therapy? When asked to rate perceived amount of change following $\mathrm{T}$ therapy, voice masculinity was rated as the most changed among the surveyed traits with $72 \%$ of participants indicating that voice masculinity was " 6 " or a " 7 " on the 1-to-7 scale (7 indicated an enormous amount 
A Happiness with traits prior to transition

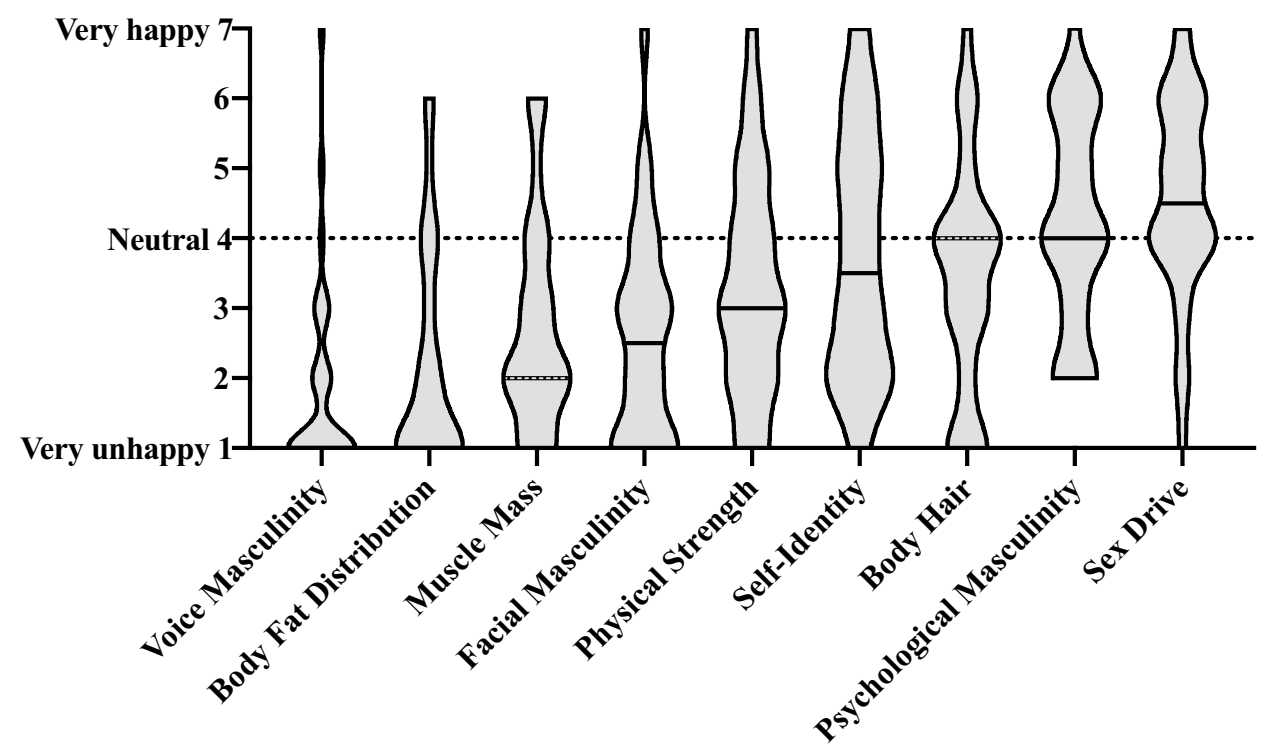

$\mathbf{B}$

Importance of trait changing with testoster one therapy

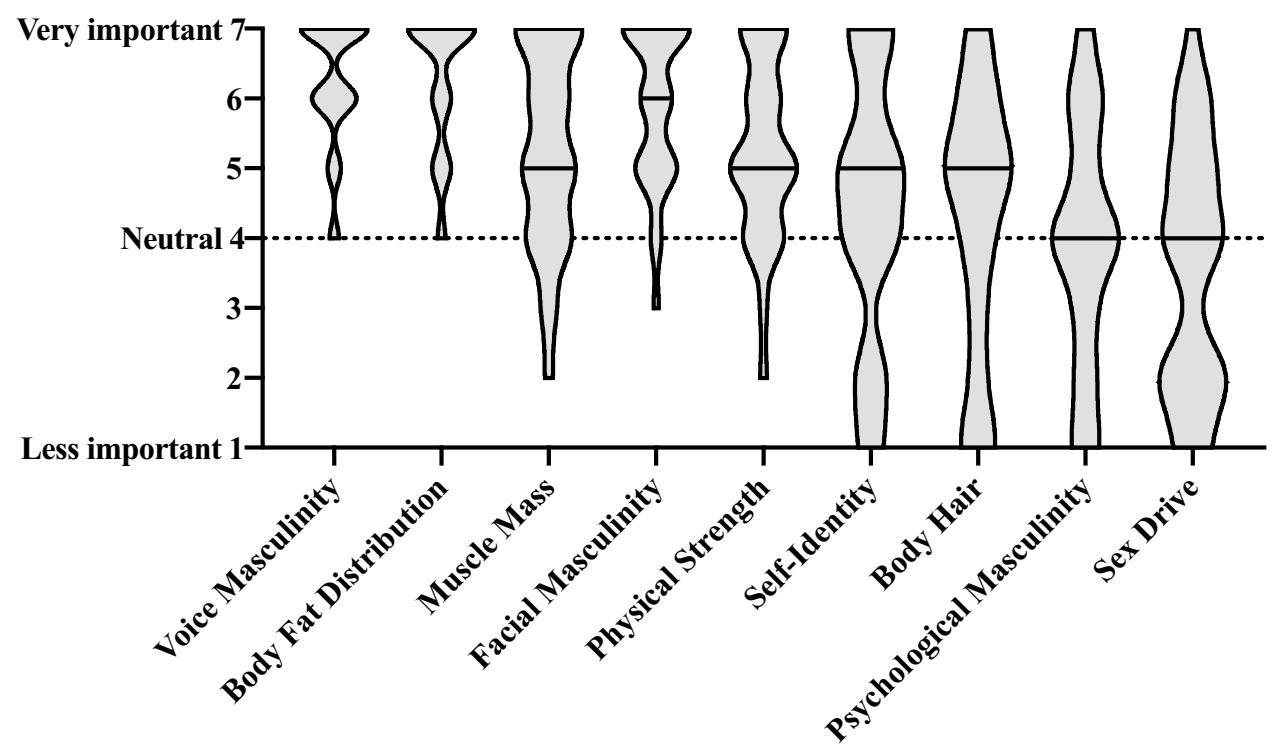

Figure 1. Satisfaction with traits prior to testosterone therapy (A) and importance of trait changing with testosterone therapy (B).

\begin{tabular}{|l|l|l|l|}
\hline & $\boldsymbol{f}_{\mathbf{o}}(\mathbf{H z})$ & $\boldsymbol{f}_{\mathbf{o}}-\mathrm{SD}(\mathrm{Hz})$ & VTL $(\mathbf{c m})$ \\
\hline \multirow{2}{*}{$\begin{array}{l}\text { Transmen } \\
(\mathrm{N}=30)\end{array}$} & $116.8( \pm 16.9)$ & $17.8( \pm 7.4)$ & $15.7( \pm 0.8)$ \\
\cline { 2 - 4 } & $93.7-150.9$ & $7.8-36.3$ & $14.3-17.2$ \\
\hline $\begin{array}{l}\text { Cismen } \\
(\mathrm{N}=34)\end{array}$ & $110.6( \pm 16.6)$ & $13.9( \pm 4.8)$ & $16.9( \pm 0.9)$ \\
\cline { 2 - 4 } & $89.5-151.8$ & $6.9-23.0$ & $15.3-19.0$ \\
\hline $\begin{array}{l}\text { Ciswomen } \\
(\mathrm{N}=32)\end{array}$ & $192.5( \pm 25.7)$ & $32.9( \pm 11.8)$ & $15.0( \pm 0.7)$ \\
\cline { 2 - 4 } & $133.4-238.6$ & $12.6-54.1$ & $13.7-16.6$ \\
\hline
\end{tabular}

Table 1. Descriptive statistics for speech characteristics: Mean $( \pm \mathrm{SD})$ and range. $f_{\mathrm{o}}$ and $f_{\mathrm{o}}-\mathrm{SD}$ measured from the Rainbow Passage. VTL estimated from vowels $/ \varepsilon /$ and $/ \mathrm{D} /$. 

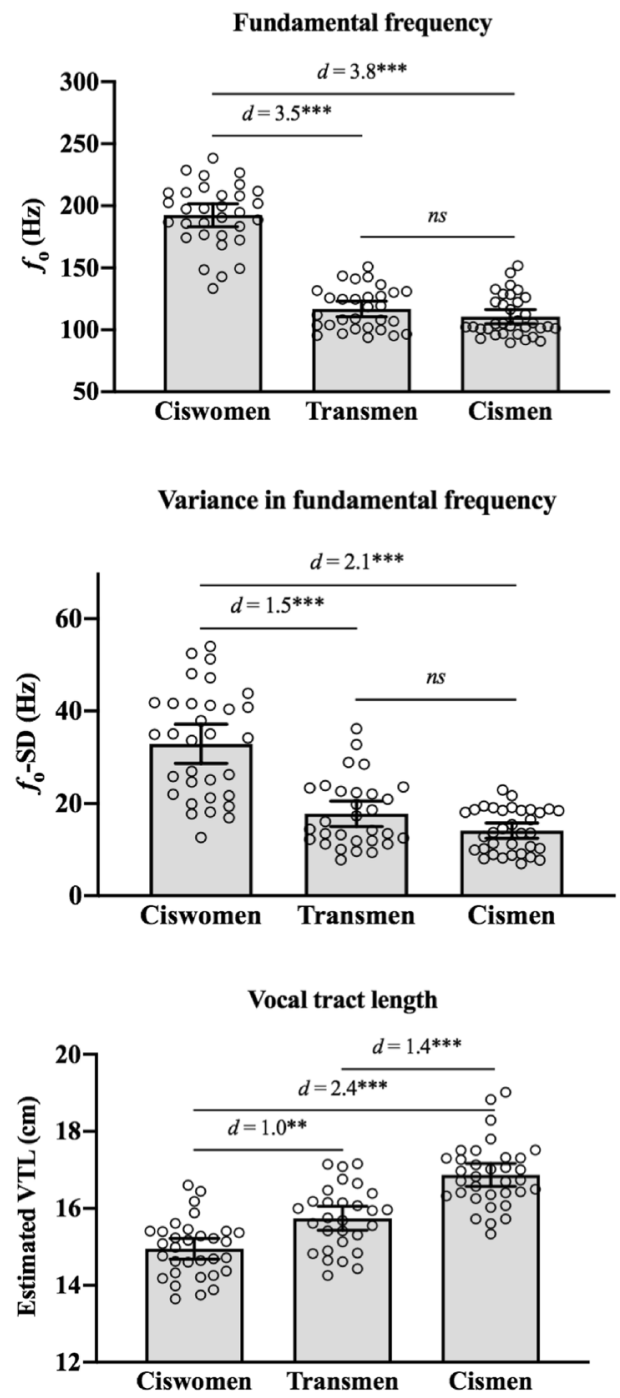

Figure 2. Fundamental frequency mean $\left(f_{\mathrm{o}}\right)$ and variation $\left(f_{\mathrm{o}}-\mathrm{SD}\right)$, and estimated vocal tract length (VTL) in ciswomen, transmen, and cismen speakers. Note: ${ }^{\star \star} p<.01 ;{ }^{\star * \star} p<.001$. Fundamental frequency mean and variation were from the Rainbow Passage and vocal tract length was estimated from vowels $/ \varepsilon /$ and $/ \mathrm{D} /$. Error bars represent $95 \%$ confidence intervals.

of change observed in the trait). When asked to rate satisfaction with perceived changes, voice masculinity was rated the highest among all survey traits with $77 \%$ indicating a " 1 " or a " 2 " ( 1 indicated that they were extremely satisfied with the changes). See Fig. 5.

\section{Discussion}

Voice masculinization is particularly important to transgender individuals undergoing a female-to-male transition; compared with eight other masculinity traits, participants indicated that they were least satisfied with their voice prior to transition and ranked it highest in priority for seeing change. Further, after T therapy (which was effective at masculinizing $f_{\mathrm{o}}$ and $f_{\mathrm{o}}$-SD in our participants), transmen were most satisfied with their vocal masculinization compared with other traits. Given its importance in a female-to-male transition and the growing number of individuals undertaking this treatment ${ }^{46,47}$, the need for evidence-based research on voice masculinization is high.

Our results show that, on average, T therapy is effective at masculinizing $f_{\mathrm{o}}$ and $f_{\mathrm{o}}$-SD. Transmen's $f_{\mathrm{o}}$ values (mean and range) are comparable to those of cismen and statistically significantly lower than ciswomen's $f_{\mathrm{o}}$ values. While we do not have recordings of these men prior to T therapy, we can assume that their $f_{\mathrm{o}}$ was close to the average for ciswomen and that their $f_{\mathrm{o}}$ has since changed by 3.5 standard deviations (or more ${ }^{28}$ ), which is nearly $80 \mathrm{~Hz}$. Research suggests that $50 \%$ of listeners can detect shifts as low as 1.2 semitones (e.g., $7 \mathrm{~Hz}$ for a $100 \mathrm{~Hz}$ voice ${ }^{42}$ ); therefore, these changes likely have a strong impact on perception of gender. Overall, the current findings are consistent with previous results documenting substantial changes in $f_{\mathrm{o}}$ with T therapy in transmen ${ }^{32-36}$ and are suggestive of putative anatomical changes resulting from the action of $\mathrm{T}$ on the lengthening and thickening of vocal folds, similar to those occurring during puberty in natal males ${ }^{19-24}$. To understand the nature of these 


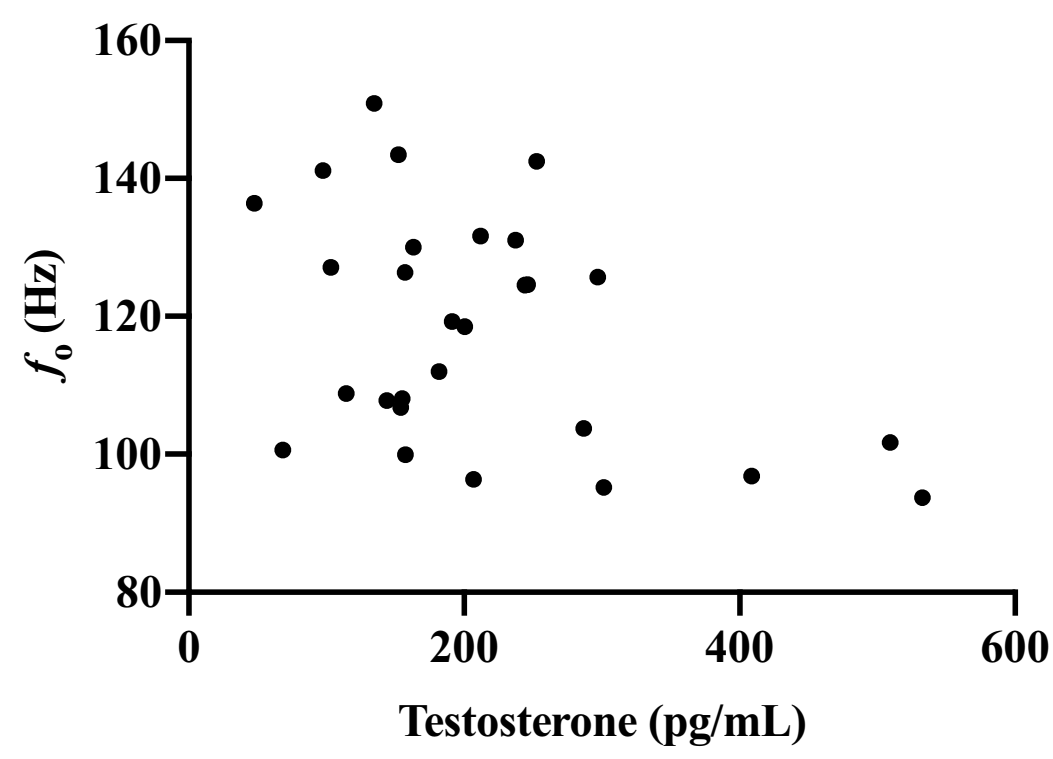

Figure 3. Association between salivary testosterone and fundamental frequency $\left(f_{\mathrm{o}}\right)$.

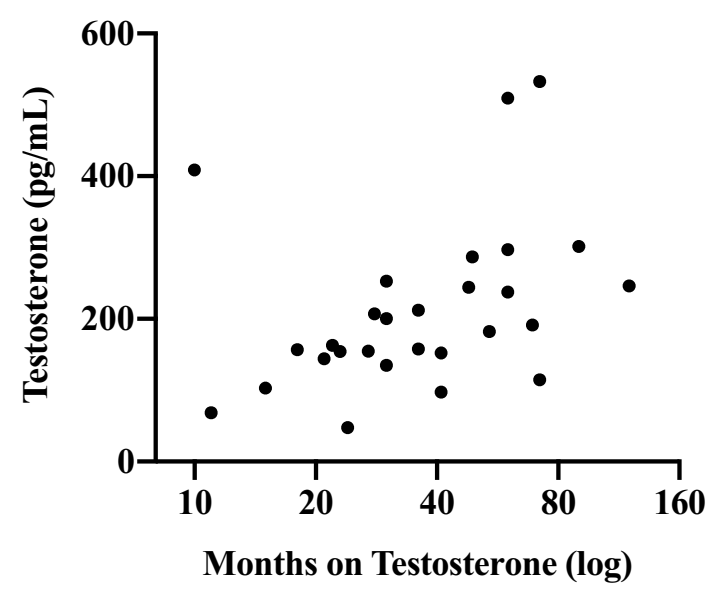

Figure 4. Salivary testosterone levels in transmen by time on $\mathrm{T}$ therapy.

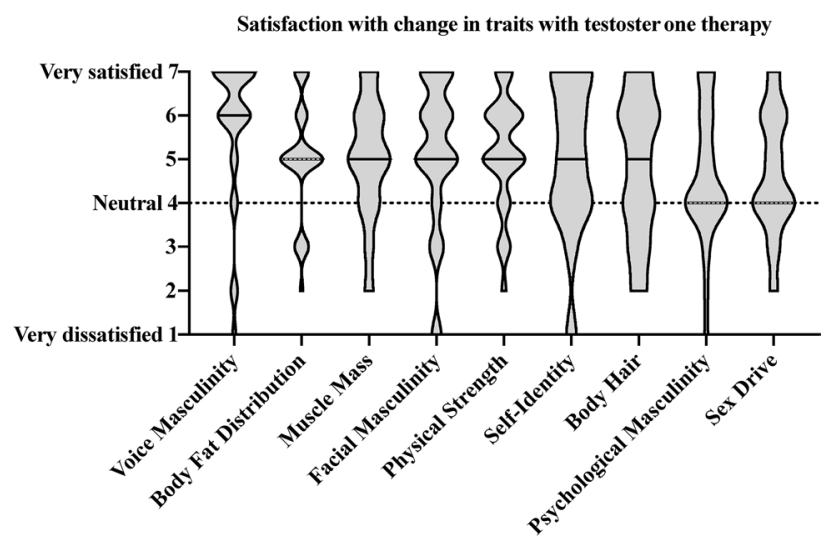

Figure 5. Satisfaction with perceived change in traits following testosterone therapy. 
structural changes, future studies should use imaging techniques to objectively quantify vocal fold length and thickness at regular intervals during $\mathrm{T}$ therapy.

$\mathrm{T}$ therapy may not be sufficient for achieving formant frequencies that are indistinguishable from cismen. Our results showed that transmen's estimated VTL was significantly longer than ciswomen but shorter than cismen. $23 \%$ of our participants' VTL fell outside the range of our cismen sample, suggesting that T therapy alone does not fully masculinize larynx position. Despite research indicating that both $f_{\mathrm{o}}$ and VTL contribute to gendered voice perception ${ }^{13-16}$, only one other published study on transmen's voice changes has examined VTL or formants ${ }^{43}$. This motivates development of additional treatments, such as behavioral therapy, to increase objective speech masculinity by increasing vocal tract length ${ }^{48}$. Previous studies on transmen's speech changes have shown that most changes have occurred prior to 9 months of continuous $\mathrm{T}$ therapy ${ }^{32-35,49}$; however, these studies did not examine changes in estimated VTL. This study is the first, to our knowledge, to demonstrate statistical differences in VTL between samples of transmen and cisgender speakers [see Cler et al. ${ }^{43}$ for a single, detailed case study and $\mathrm{Papp}^{44}$ for an unpublished dissertation].

Incomplete masculinization of VTL (as well as $f_{\mathrm{o}}-\mathrm{SD}$ ) may partly explain why $17 \%$ of our participants reported that they were 'neutral' to 'extremely dissatisfied' with changes in their vocal masculinity. This accords with previous studies showing $12-16 \%$ of patients are not fully satisfied with their vocal transition and $25 \%$ were still sometimes perceived as female on the phone ${ }^{32,37}$. Further, $31 \%$ expressed interest in further masculinizing their speech through additional treatments like behavioral voice therapy ${ }^{32}$. Despite the need for behavioral voice therapy among transmen, only one published study has examined its efficacy ${ }^{48}$. This is in contrast to transfeminine individuals where it has been shown to help individuals express their gender identity through speech, reduce gender dysphoria, and improve mental health and quality of life $\mathrm{e}^{50-53}$.

Some of the acoustic properties of speech that drive gender perception were associated with features of $\mathrm{T}$ therapy. We found a significant inverse association between salivary $\mathrm{T}$ and $f_{\mathrm{o}}$; however, the results appear largely driven by 3 data points (see Fig. 3). Given the small sample size, it is unclear whether these individuals represent the normal range of variation. The association between current salivary $\mathrm{T}$ and $f_{\mathrm{o}}$ makes theoretical sense given the longer-term association between $\mathrm{T}$ administration and $f_{\mathrm{o}}$ change in transmen, the presence of androgen receptors on the vocal folds ${ }^{54,55}$, and the associations between $\mathrm{T}$ and $f_{\mathrm{o}}$ during puberty ${ }^{18-23}$. In addition, several studies have found links between salivary T and masculine vocal parameters in cismen ${ }^{25,26,28}$ ( $\mathrm{cf}$. Arnocky et al. ${ }^{29}$ ), and one study of cismen showed within-individual diurnal decreases in salivary T were associated with increases in $f_{\mathrm{o}}{ }^{27}$. Given the strong empirical and theoretical support for an association between $\mathrm{T}$ and $f_{\mathrm{o}}$, it is surprising that two previous studies on female-to-male speech changes ${ }^{35,36}$ did not find an association between serum T levels and $f_{\mathrm{o}}$.

Although there was not a significant association between salivary T and estimated VTL, T therapy duration was statistically significantly associated with VTL: longer T therapy durations were associated with longer estimated VTLs. This finding may suggest a longer-term relationship between T therapy and VTL. However, an alternative explanation is that this association reflects the confounding effect of time since transition given its close association with duration of $\mathrm{T}$ therapy. That is, even without formal voice training, transmen may be implicitly learning how to manipulate their vocal tract over time to achieve longer VTLs. Clinical studies on the relationships among dosing regimen, biological $\mathrm{T}$ availability, and speech parameters among transmen are necessary.

In summary, we see two important implications of these findings. First, a voice with a low pitch is a central aspect of masculine gender presentation because it is easily observable, highly sexually dimorphic, and difficult to approximate if not an adult male. Vocal fold dimorphism is one of the largest anatomical sex differences observed in humans (approximately 5 standard deviations ${ }^{28,56}$ ) and greater than any other extant ape $\mathrm{e}^{57}$. Cisgender men and women differ by $60 \%$ in vocal fold length ${ }^{30}$ but only $8 \%$ in height ${ }^{58}$. Because vocal sexual dimorphism is extensive and, importantly, features little overlap in gender-typical vocal ranges, it is extremely difficult to speak in a voice consistent with the opposite sex, particularly in a sustained fashion ${ }^{31}$. These facts help explain why transmen are so dissatisfied with their $f_{\mathrm{o}}$ prior to T therapy. Similarly, our participants were also highly dissatisfied with body fat distribution, which is also very dimorphic ${ }^{58,59}$, easily observable, and difficult to change without hormonal therapy.

A second implication of these findings is that more research on speech changes in transgender males is necessary. The studies that have been published are limited by small sample sizes ${ }^{32-34,49}$, a lack of a control group for comparisons $s^{32,33,35}$, and a focus on only $f_{\mathrm{o}}^{34,49}$. Additional research on $\mathrm{T}$ dosing regimens as well as the efficacy of behavioral voice therapy are particularly necessary. Better evidence-based treatments for transmen have health and safety repercussions. Transgender individuals are disproportionately targets of violence and being viewed as one's gender is likely a critical component for safety ${ }^{60-63}$. Approximately $20-47 \%$ of transgender individuals have been physically or sexually assaulted and an additional $34-46 \%$ have been verbally threatened or harassed ${ }^{62,64}$.

In contrast to voice masculinization, participants did not place high importance on seeing an effect of $\mathrm{T}$ therapy on the non-physical trait "psychological masculinity", highlighting participants' dissociation between their own perception of gender and outward display of gender prior to therapy ${ }^{65}$. This incongruence is a source of extreme distress, which is associated with higher levels of depression, anxiety, substance abuse, and suicidal ideation and attempts among the transgender population-particularly those that have not begun to transition ${ }^{5,7,9,65-68}$. Receiving hormone treatment significantly improves mental health, social health, and physical health outcomes in transgender populations $s^{2,7,9,66}$. Vocal congruence contributes to these improvements; Watt et al. ${ }^{2}$ showed that more masculine voices significantly contributed to improved well-being and mental health in female-to-male transgender patients.

To summarize, this research was designed with several goals in mind. First, we aimed to quantify the importance of voice change-relative to other masculine traits-for transgender men undergoing testosterone therapy. No previous studies have explored this question, in spite of the strong interest in voice change among the transmasculine population. Our results show that voice masculinization is of central importance to transgender individuals undergoing the female-to-male transition compared with eight other masculine traits. Second, we 
asked whether $\mathrm{T}$ therapy was effective at masculinizing three gendered speech parameters. Our results show that, on average, T therapy is effective at masculinizing fundamental frequency mean and variation $\left(f_{\mathrm{o}}\right.$ and $f_{\mathrm{o}}$-SD); however, transmen's formant-based measure of vocal tract length (VTL) was significantly shorter than cismen. This study is the first, to our knowledge, to demonstrate statistical differences in VTL between samples of transmen and cisgender speakers. Third, we examined the association between salivary testosterone and vocal parameters. We found a significant inverse association between salivary $\mathrm{T}$ and fundamental frequency but no association with VTL. T therapy duration, however, was statistically significantly associated with VTL. These findings point to the need for more research on speech changes in transgender males-of particular importance are transition strategies that affect formant frequencies, which have largely been ignored in previous research.

\section{Method}

Participants. Transgender men. Participants included 30 individuals who were assigned female at birth; four of the participants identified their gender as non-binary and 26 identified as men. All were currently undergoing hormone replacement therapy with $\mathrm{T}$ for at least 9 months $\left(M_{\text {months }}=41.50, S D=25.45\right)$ for the purpose of masculinization. Administration routes varied (intramuscular $=13$, every $7-14$ days; subcutaneous $=11$, every 5-14 days; subdermal pellets $=4$, every 3-4 months; transdermal $=1$, daily). Ages ranged from 20-40 years old (mean: 25.9yrs \pm 5.2 ) and the age when T therapy began ranged from 17 to 38 years old (mean: 22.4yrs \pm 5.2 ); these were closely correlated with each other $(r=0.92, p<0.001)$, but neither correlated significantly with time on $\mathrm{T}$ therapy or salivary T. Participants reported being primarily attracted to men $(\mathrm{N}=6)$, women $(\mathrm{N}=7)$, or both men and women $(\mathrm{N}=15)$. Two participants declined to answer. Research suggests that lesbian women have lower $f_{\mathrm{o}}$ and $f_{\mathrm{o}}$-SD compared to heterosexual women (van Borsel et al., 2017); however, our sample size prohibited analysis by sexual orientation. Participants were excluded if they had been diagnosed with any vocal pathology or if they were habitual smokers in order to minimize the effect of potential spurious relationships. The ethnic composition of the sample was: Caucasian (86.7\%), Asian (3.3\%), Latin American (3.3\%) and multiple ethnicities (3.3\%). Recruitment was conducted via digital flyers and notifications on private or closed LGBTQ + or transgender Facebook groups, physical flyers posted at the 2017 Boston Pride festival, and word of mouth.

Cisgender men. Voice samples were collected from 122 cisgender male individuals. From this sample, we selected all individuals aged 21 and older to bring the mean age closer to that of the trans sample. The final $\mathrm{N}$ for cisgender men was 34. Ages ranged from 21 to 28 years old (mean: 23.0 years \pm 2.2 ). More detail on this sample can be found in Arnocky et al. ${ }^{29}$.

Cisgender women. Participants included 32 undergraduate females from a larger study on immune function and phenotypic characteristics. From the larger data set, we selected all individuals 21 and older. Ages ranged from 21 to 32 years old (mean: 22.6 years \pm 2.8 ). The sample was comprised primarily of Caucasian $(94 \%)$ women.

Procedure. All procedures were approved by the Boston University Institutional Review Board or the Nipissing University Research Ethics Board and were performed in accordance with relevant guidelines and regulations. Informed consent was obtained from all participants.

Questionnaire. Transgender participants answered a survey covering the following topics (7-point Likert scales, $4=$ neutral): satisfaction with nine physical and/or psychological characteristics (see below) prior to transition ( $1=$ I was very unhappy; $7=I$ was very happy), importance of seeing changes in those characteristics during transition $(1=$ Changes were not important to $m e ; 7=$ Changes were very important to $m e)$, amount of observed change in those characteristics since starting $\mathrm{T}$ therapy $(1=$ No change at all; $7=$ Enormous amount of change $)$, satisfaction with this change since starting $\mathrm{T}$ therapy $(1=I$ am very unhappy; $7=I$ am very happy), and overall satisfaction with their medical transition $(1=$ I am very unhappy; $7=$ I am very happy). The surveyed characteristics were voice masculinity, facial masculinity (excluding facial hair), body hair, amount of muscle mass, physical strength, psychological masculinity, self-identity, sex drive, and body fat distribution.

Voice Recording. All speakers were asked to recite, in their normal speaking voice, the first sentence of the Rainbow Passage, numbers 1 to 10, and vowel sounds in the order / $/$ / /i/ / $/ / / o \mho / / u /$. Cis- and transmen's voice samples were collected in a quiet room using an Audio-Technica AT4041 Cardioid Condenser Microphone connected to a Focusrite Scarlett $2 \mathrm{i} 2$ audio interface.

For the ciswomen sample, participants were asked to recite the same vowel sounds in the identical order described above. Voice samples were recorded in a sound attenuated room using a Neewer NW-700 condenser microphone with a $48 \mathrm{~V}$ phantom power supply and a pop filter. Participant voices were recorded using Goldwave version 6.31 software in mono with a sampling rate of $44.1 \mathrm{kHz}$ and 16-bit quantization. The voice recordings were saved as high quality uncompressed wav files.

For all samples, recordings were analyzed using Praat version $5.3^{69}$ for mean $f_{\mathrm{o}}$ and standard deviation in $f_{\mathrm{o}}$ across the utterance $\left(f_{\mathrm{o}}-\mathrm{SD}\right)$ using the 'voice report' function. Pitch floor was set to $75 \mathrm{~Hz}$ and pitch ceiling was set to $300 \mathrm{~Hz}$ for cismen and transmen, and $100 \mathrm{~Hz}$ and $500 \mathrm{~Hz}$ for ciswomen, which are the recommended parameters for men's and women's voices, respectively. Otherwise, default settings were used. We used the Rainbow Passage for analyses involving $f_{\mathrm{o}}$ and $f_{\mathrm{o}}$-SD; however, between-group comparisons (i.e., transmen vs. cismen and transmen vs. ciswomen) were similar when either vowels or counting were used for analyses. We also computed 
$f_{o}$-CV $\left(f_{\mathrm{o}}\right.$-SD / mean $\left.f_{\mathrm{o}}\right)$ following Pisanski et al. ${ }^{70}$ Transmen were not significantly different from either ciswomen or cismen in $f_{o}-\mathrm{CV}$; therefore, we only report $f_{\mathrm{o}}$-SD below.

In order to estimate VTL, formants were calculated using the acoustic recordings of the vowel sounds $/ \varepsilon /$ and $/ \mathrm{D} /$. All other vowels were omitted from VTL estimates due to narrow constrictions of the vocal tract $(/ \mathrm{i} /)$ or lip rounding $(/ \mathrm{o} / /$ and $/ \mathrm{u} /)$ that complicate the relationship between VTL and formant frequencies ${ }^{71}$. Praat was used to generate a wide-band spectrogram of the acoustic signal, which was then used to calculate the first four formants using the standard formant tracking software in Praat. For each vowel, these automated formants were visually inspected, and the settings of the tracking software were adjusted until the formants aligned with the spectral representation of the signal. Formant values were calculated over the central stable part of the vowel. Third (F3) and fourth (F4) formant values for each participant were calculated by averaging the values from both vowels. These formant values were then used to calculate VTL estimates via Eq. $(1)^{72}$, which shows an inverse linear relationship between formants and VTL that is derived from modeling the vocal tract as a uniform tube that is closed at one end (i.e., the vocal folds) and open at the other (i.e., the mouth). In Eq. (1), $n$ is the formant number, $\mathrm{F}_{n}$ is the formant frequency (in $\mathrm{Hz}$ ), and $c$ is the speed of sound. Finally, VTL estimates from F3 and F4 are averaged for a single VTL estimate for each participant. F3 and F4 are used, because higher formants tend to be more stable and a better estimate of $\mathrm{VTL}^{73}$.

$$
\mathrm{VTL}=\frac{(2 \mathrm{n}-1) \times \mathrm{c}}{4 \times \mathrm{F}_{\mathrm{n}}}
$$

Saliva collection and analysis. Participants collected saliva samples in a 2-mL cryovial immediately upon waking the morning after they visited the lab to provide questionnaires and voice samples. Mean sample provision start time was 8:44 AM $\pm 1 \mathrm{~h} 59 \mathrm{~min}$. Samples were refrigerated immediately after collection and then brought to the research team the following day where they were inspected using the Blood Contamination in Saliva Scale ${ }^{74}$. They were then stored in a $-80^{\circ} \mathrm{C}$ freezer until they were shipped overnight on dry ice for analysis. Samples were assayed in duplicate for free $\mathrm{T}$ using commercially available enzyme-linked immunoassay kits (DRG International, NJ, USA); average intra- and inter-assay coefficients of variation were $6.84 \%$ and $8.97 \%$, respectively. For further detail on collection and assay of saliva samples, see Arnocky et al. ${ }^{29}$. T means and variance were significantly higher in transmen $(\mathrm{M}=321.3 \mathrm{pg} / \mathrm{mL}, \mathrm{SD}=509, \mathrm{MIN}=47.7, \mathrm{MAX}=2889)$; however, when two outliers were removed $(\mathrm{T}=784$ and $2889 \mathrm{pg} / \mathrm{mL})$, the trans mean $(\mathrm{M}=212.8 \mathrm{pg} / \mathrm{mL}, \mathrm{SD}=116.8, \mathrm{MIN}=47.7$, $\mathrm{MAX}=538.2)$ was closer to the expected physiological range, compared with published averages for cismen $\left(\mathrm{M}=152.1 \mathrm{pg} / \mathrm{mL}, \mathrm{SD}=60.5, \mathrm{MIN}=28.7, \mathrm{MAX}=152.0\right.$; values from Arnocky et al.$\left.^{29}\right)$.

Data analysis. The following variables were log-natural transformed to address skew and increase normality: salivary $\mathrm{T}$, time on $\mathrm{T}$ therapy, and $f_{\mathrm{o}}$. A univariate analysis of variance (ANOVA) was used to compare groups (transmen, cismen, and ciswomen) for each of the speech parameters $\left(f_{\mathrm{o}}, f_{\mathrm{o}}\right.$-SD, and VTL). Initially, age was added to the model as a covariate and was then removed because it did not affect the analysis outcome. Linear regressions were used to examine associations between salivary $\mathrm{T}$ and speech parameters in transmen.

Received: 5 June 2020; Accepted: 9 December 2020

Published online: 10 February 2021

\section{References}

1. Aitken, M., VanderLaan, D. P., Wasserman, L., Stojanovski, S. \& Zucker, K. J. Self-harm and suicidality in children referred for gender dysphoria. J. Am. Acad. Child Adolesc. Psychiatry 55, 513-520 (2016).

2. Watt, S. O., Tskhay, K. O. \& Rule, N. O. Masculine voices predict well-being in female-to-male transgender individuals. Arch. Sex. Behav. 47, 963-972 (2018).

3. Zucker, K. J., Lawrence, A. A. \& Kreukels, B. P. Gender dysphoria in adults. Annu. Rev. Clin. Psychol. 12, 217-247 (2016).

4. Clements-Nolle, K., Marx, R. \& Katz, M. Attempted suicide among transgender persons: the influence of gender-based discrimination and victimization. J. Homosex. 51, 53-69 (2006).

5. Bariola, E. et al. Demographic and psychosocial factors associated with psychological distress and resilience among transgender individuals. Am. J. Public Health 105, 2108-2116 (2015).

6. Dhejne, C., Van Vlerken, R., Heylens, G. \& Arcelus, J. Mental health and gender dysphoria: a review of the literature. Int. Rev. Psychiatry 28, 44-57 (2016).

7. Budge, S. L., Adelson, J. L. \& Howard, K. A. Anxiety and depression in transgender individuals: the roles of transition status, loss, social support, and coping. J. Consult. Clin. Psychol. 81, 545 (2013).

8. Factor, R. J. \& Rothblum, E. D. A study of transgender adults and their non-transgender siblings on demographic characteristics, social support, and experiences of violence. J. LGBT Health Res. 3, 11-30 (2007).

9. Davis, S. A. \& Colton Meier, S. Effects of testosterone treatment and chest reconstruction surgery on mental health and sexuality in female-to-male transgender people. Int. J. Sex. Health 26, 113-128 (2014).

10. Costa, R. \& Colizzi, M. The effect of cross-sex hormonal treatment on gender dysphoria individuals' mental health: a systematic review. Neuropsychiatr. Dis. Treat. 12, 1953 (2016).

11. Heylens, G. et al. Psychiatric characteristics in transsexual individuals: multicentre study in four European countries. Br. J. Psychiatry 204, 151-156 (2014).

12. Oda, H. \& Kinoshita, T. Efficacy of hormonal and mental treatments with MMPI in FtM individuals: cross-sectional and longitudinal studies. BMC Psychiatry 17, 256 (2017).

13. Assmann, P. F., Nearey, T. M. \& Chen, D. Matching fundamental and formant frequencies in vowels. J. Acoust. Soc. Am. 120, 3248-3248 (2006)

14. Bachorowski, J.-A. \& Owren, M. J. Acoustic correlates of talker sex and individual talker identity are present in a short vowel segment produced in running speech. J. Acoust. Soc. Am. 106, 1054-1063 (1999).

15. Hillenbrand, J. M. \& Clark, M. J. The role of $\mathrm{f} 0$ and formant frequencies in distinguishing the voices of men and women. Atten. Percept. Psychophys. 71, 1150-1166 (2009). 
16. Smith, D. R. \& Patterson, R. D. The interaction of glottal-pulse rate and vocal-tract length in judgements of speaker size, sex, and age. J. Acoust. Soc. Am. 118, 3177-3186 (2005).

17. Fitch, W. T. \& Giedd, J. Morphology and development of the human vocal tract: a study using magnetic resonance imaging. J. Acoust. Soc. Am. 106, 1511-1522 (1999).

18. Butler, G. et al. Salivary testosterone levels and the progress of puberty in the normal boy. Clin. Endocrinol. (Oxf.) 30, 587-596 (1989).

19. Harries, M. L. L., Walker, J. M., Williams, D. M., Hawkins, S. \& Hughes, I. A. Changes in the male voice at puberty. Arch. Dis. Child. 77, 445-447 (1997).

20. Harries, M., Hawkins, S., Hacking, J. \& Hughes, I. Changes in the male voice at puberty: vocal fold length and its relationship to the fundamental frequency of the voice. J. Laryngol. Otol. 112, 451-454 (1998).

21. Hodges-Simeon, C. R., Gurven, M., Cárdenas, R. A. \& Gaulin, S. J. Voice change as a new measure of male pubertal timing: a study among Bolivian adolescents. Ann. Hum. Biol. 40, 209-219 (2013).

22. Hodges-Simeon, C. R., Gurven, M. \& Gaulin, S. J. C. The low male voice is a costly signal of phenotypic quality among Bolivian adolescents. Evol. Hum. Behav. 36, 294-302 (2015).

23. Hollien, H., Green, R. \& Massey, K. Longitudinal research on adolescent voice change in males. J. Acoust. Soc. Am. 96, 2646-2654 (1994).

24. Markova, D. et al. Age-and sex-related variations in vocal-tract morphology and voice acoustics during adolescence. Horm. Behav. 81, 84-96 (2016).

25. Aung, T. \& Puts, D. Voice pitch: a window into the communication of social power. Curr. Opin. Psychol. 33, 154-161 (2020).

26. Dabbs, J. M. Jr. \& Mallinger, A. High testosterone levels predict low voice pitch among men. Personal. Individ. Differ. 27, 801-804 (1999).

27. Evans, S., Neave, N., Wakelin, D. \& Hamilton, C. The relationship between testosterone and vocal frequencies in human males. Physiol. Behav. 93, 783-788 (2008).

28. Puts, D. A., Apicella, C. L. \& Cárdenas, R. A. Masculine voices signal men’s threat potential in forager and industrial societies. Proc. R. Soc. B Biol. Sci. 279, 601-609 (2012).

29. Arnocky, S., Hodges-Simeon, C. R., Ouellette, D. \& Albert, G. Do men with more masculine voices have better immunocompetence?. Evol. Hum. Behav. 39, 602-610 (2018).

30. Titze, I. R. Principles of Voice Production (Second Printing) (Iowa City IA Natl. Cent, Voice Speech, 2000).

31. Davies, S., Papp, V. G. \& Antoni, C. Voice and communication change for gender nonconforming individuals: giving voice to the person inside. Int. J. Transgenderism 16, 117-159 (2015).

32. Van Borsel, J., De Cuypere, G., Rubens, R. \& Destaerke, B. Voice problems in female-to-male transsexuals. Int. J. Lang. Commun. Disord. 35, 427-442 (2000).

33. Damrose, E. J. Quantifying the impact of androgen therapy on the female larynx. Auris Nasus Larynx 36, 110-112 (2009).

34. Deuster, D. et al. Voice deepening under testosterone treatment in female-to-male gender dysphoric individuals. Eur. Arch. Otorhinolaryngol. 273, 959-965 (2016).

35. Nygren, U., Nordenskjöld, A., Arver, S. \& Södersten, M. Effects on voice fundamental frequency and satisfaction with voice in trans men during testosterone treatment-a longitudinal study. J. Voice 30, 766-e23 (2016).

36. Cosyns, M. et al. Voice in female-to-male transsexual persons after long-term androgen therapy. The Laryngoscope 124, 1409-1414 (2014).

37. Ziegler, A., Henke, T., Wiedrick, J. \& Helou, L. B. Effectiveness of testosterone therapy for masculinizing voice in transgender patients: a meta-analytic review. Int. J. Transgenderism 19, 25-45 (2018).

38. Nakamura, A. et al. Dose-response analysis of testosterone replacement therapy in patients with female to male gender identity disorder. Endocr. J. EJ12-0319 (2012).

39. Gelfer, M. P. \& Mikos, V. A. The relative contributions of speaking fundamental frequency and formant frequencies to gender identification based on isolated vowels. J. Voice 19, 544-554 (2005).

40. Skuk, V. G. \& Schweinberger, S. R. Influences of fundamental frequency, formant frequencies, aperiodicity, and spectrum level on the perception of voice gender. J. Speech Lang. Hear. Res. 57, 285 (2014).

41. Gelfer, M. P. \& Bennett, Q. E. Speaking fundamental frequency and vowel formant frequencies: effects on perception of gender. J. Voice 27, 556-566 (2013).

42. Puts, D. A., Hodges, C. R., Cárdenas, R. A. \& Gaulin, S. J. Men’s voices as dominance signals: vocal fundamental and formant frequencies influence dominance attributions among men. Evol. Hum. Behav. 28, 340-344 (2007).

43. Cler, G. J., McKenna, V. S., Dahl, K. L. \& Stepp, C. E. Longitudinal case study of transgender voice changes under testosterone hormone therapy. J. Voice https://doi.org/10.1016/j.jvoice.2019.03.006 (2019).

44. Papp, V. The female-to-male transsexual voice: Physiology vs. performance in production. (2012).

45. Grubbs, F. E. Procedures for detecting outlying observations in samples. Technometrics 11, 1-21 (1969).

46. Clark, T. C. et al. The health and well-being of transgender high school students: results from the New Zealand adolescent health survey (Youth'12). J. Adolesc. Health 55, 93-99 (2014).

47. Flores, A., Herman, J., Gates, G. \& Brown, T. How Many Adults Identify as Transgender in the United States? Los Angeles (The Williams Institute, CA, 2016).

48. Buckley, D. P., Dahl, K. L., Cler, G. J. \& Stepp, C. E. Transmasculine voice modification: a case study. J. Voice https://doi. org/10.1016/j.jvoice.2019.05.003 (2019).

49. Irwig, M. S., Childs, K. \& Hancock, A. B. Effects of testosterone on the transgender male voice. Andrology 5, 107-112 (2017).

50. Carew, L., Dacakis, G. \& Oates, J. The effectiveness of oral resonance therapy on the perception of femininity of voice in male-tofemale transsexuals. J. Voice 21, 591-603 (2007).

51. Dacakis, G., Oates, J. \& Douglas, J. Beyond voice: perceptions of gender in male-to-female transsexuals. Curr. Opin. Otolaryngol. Head Neck Surg. 20, 165-170 (2012).

52. Gelfer, M. P. \& Tice, R. M. Perceptual and acoustic outcomes of voice therapy for male-to-female transgender individuals immediately after therapy and 15 months later. J. Voice 27, 335-347 (2013).

53. Hancock, A. B. \& Garabedian, L. M. Transgender voice and communication treatment: a retrospective chart review of 25 cases. Int. J. Lang. Commun. Disord. 48, 54-65 (2013).

54. Newman, S.-R., Butler, J., Hammond, E. H. \& Gray, S. D. Preliminary report on hormone receptors in the human vocal fold. J. Voice 14, 72-81 (2000).

55. Voelter, C. et al. Detection of hormone receptors in the human vocal fold. Eur. Arch. Otorhinolaryngol. 265, 1239-1244 (2008).

56. Rendall, D., Kollias, S., Ney, C. \& Lloyd, P. Pitch (F 0) and formant profiles of human vowels and vowel-like baboon grunts: The role of vocalizer body size and voice-acoustic allometry. J. Acoust. Soc. Am. 117, 944-955 (2005).

57. Puts, D. A. et al. Sexual selection on male vocal fundamental frequency in humans and other anthropoids. Proc. R. Soc. B Biol. Sci. 283, 20152830 (2016).

58. Lassek, W. D. \& Gaulin, S. J. Costs and benefits of fat-free muscle mass in men: Relationship to mating success, dietary requirements, and native immunity. Evol. Hum. Behav. 30, 322-328 (2009).

59. Wells, J. C. Sexual dimorphism of body composition. Best Pract. Res. Clin. Endocrinol. Metab. 21, 415-430 (2007). 
60. Coleman, E. et al. Standards of care for the health of transsexual, transgender, and gender-nonconforming people, version 7. Int. J. Transgenderism 13, 165-232 (2012).

61. Hancock, A. B., Krissinger, J. \& Owen, K. Voice perceptions and quality of life of transgender people. J. Voice 25, 553-558 (2011).

62. James, S. et al. The report of the 2015 US transgender survey. (2016).

63. Safer, J. D. et al. Barriers to health care for transgender individuals. Curr. Opin. Endocrinol. Diabetes Obes. 23, 168 (2016).

64. Bauer, G. \& Scheim, A. Transgender people in Ontario, Canada: Statistics from the Trans PULSE Project to inform human rights policy. (Trans Pulse, 2015).

65. Olson, J., Schrager, S. M., Belzer, M., Simons, L. K. \& Clark, L. F. Baseline physiologic and psychosocial characteristics of transgender youth seeking care for gender dysphoria. J. Adolesc. Health 57, 374-380 (2015).

66. Colton Meier, S. L., Fitzgerald, K. M., Pardo, S. T. \& Babcock, J. The effects of hormonal gender affirmation treatment on mental health in female-to-male transsexuals. J. Gay Lesbian Ment. Health 15, 281-299 (2011).

67. Durwood, L., McLaughlin, K. A. \& Olson, K. R. Mental health and self-worth in socially transitioned transgender youth. J. Am. Acad. Child Adolesc. Psychiatry 56, 116-123 (2017).

68. Veale, J. F., Watson, R. J., Peter, T. \& Saewyc, E. M. Mental health disparities among Canadian transgender youth. J. Adolesc. Health 60, 44-49 (2017).

69. Boersma, P. \& Weenink, D. Praat: doing phonetics by computer 5.1. Comput. Program (2009).

70. Pisanski, K., Oleszkiewicz, A., Plachetka, J., Gmiterek, M. \& Reby, D. Voice pitch modulation in human mate choice. Proc. R. Soc. B 285, 20181634 (2018).

71. Ladefoged, P. Elements of Acoustic Phonetics (University of Chicago Press, Chicago, 1996).

72. Stevens, K. N. Acoustic Phonetics Vol. 30 (MIT press, Cambridge, 2000).

73. Wakita, H. Normalization of vowels by vocal-tract length and its application to vowel identification. IEEE Trans. Acoust. Speech Signal Process. 25, 183-192 (1977).

74. Kivlighan, K. T. et al. Quantifying blood leakage into the oral mucosa and its effects on the measurement of cortisol, dehydroepiandrosterone, and testosterone in saliva. Horm. Behav. 46, 39-46 (2004)

\section{Acknowledgments}

The authors wish to extend many thanks to the participants for their involvement with and support of the study.

\section{Author contributions}

C.R.H.-S. and G.P.O.G. Designed the study, collected and analyzed data, and drafted manuscript. GA: Data collection. M.D.G. and C.E.S. Acoustic analysis. J.C. Salivary analysis. S.A.A. Data collection. All authors: reviewed and edited the final manuscript.

\section{Funding}

Stepp and Groll: DC013017 and DC015570. Funding for the comparison sample of ciswomen was provided by a Natural Sciences and Engineering Research Council of Canada Discovery Development Grant (file \# DDG-2017-00013).

\section{Competing interests}

The authors declare no competing interests.

\section{Additional information}

Correspondence and requests for materials should be addressed to C.R.H.-S.

Reprints and permissions information is available at www.nature.com/reprints.

Publisher's note Springer Nature remains neutral with regard to jurisdictional claims in published maps and institutional affiliations.

(c) (i) Open Access This article is licensed under a Creative Commons Attribution 4.0 International License, which permits use, sharing, adaptation, distribution and reproduction in any medium or format, as long as you give appropriate credit to the original author(s) and the source, provide a link to the Creative Commons licence, and indicate if changes were made. The images or other third party material in this article are included in the article's Creative Commons licence, unless indicated otherwise in a credit line to the material. If material is not included in the article's Creative Commons licence and your intended use is not permitted by statutory regulation or exceeds the permitted use, you will need to obtain permission directly from the copyright holder. To view a copy of this licence, visit http://creativecommons.org/licenses/by/4.0/.

(C) The Author(s) 2021 